\title{
Diagnostic value of serum biomarkers FGF21 and GDF15 compared to muscle sample in mitochondrial disease
}

Jenni M. Lehtonen ${ }^{1}$, Mari Auranen ${ }^{1,2}$, Niklas Darin ${ }^{3}$, Kalliopi Sofou ${ }^{3}$, Laurence Bindoff ${ }^{4,5}$, Omar Hikmat $^{4,6}$, Johanna Uusimaa ${ }^{7}$, Päivi Vieira ${ }^{7}$, Már Tulinius ${ }^{3}$, Tuula Lönnqvist ${ }^{8}$, Irenaeus F. de $\mathrm{Coo}^{9,10}$, Anu Suomalainen ${ }^{1,11}$, Pirjo Isohanni ${ }^{1,8}$

1. Research Programs Unit, Stem Cells and Metabolism, Faculty of Medicine, University of Helsinki, Helsinki, Finland.

2. Clinical Neurosciences, Neurology, University of Helsinki and Helsinki University Hospital, Helsinki, Finland

3. Department of Pediatrics, The Queen Silvia Children's Hospital, University of Gothenburg, Gothenburg, Sweden.

4. Department of Clinical Medicine (K1), University of Bergen, Norway.

5. Department of Neurology, Haukeland University Hospital, 5021 Bergen, Norway.

6. Department of Pediatrics, Haukeland University Hospital, 5021 Bergen, Norway.

7. Department of Pediatric Neurology, Clinic for Children and Adolescents, Medical Research Center, Oulu University Hospital, and PEDEGO Research Unit, University of Oulu, Oulu, Finland. 
8. Pediatric Neurology, Children's Hospital and Pediatric Research Center, University of Helsinki and Helsinki University Hospital, Helsinki, Finland.

9. Department of Neurology, Medical Spectrum Twente, Enschede, The Netherlands.

10. Department of Genetics and Cell Biology, University of Maastricht, Maastricht, The Netherlands.

11. Neuroscience Center, HiLife, University of Helsinki, Helsinki, Finland.

\section{Correspondence to:}

Dr. Pirjo Isohanni, Children's Hospital, Stenbäckinkatu 9, 00029 HUS, Helsinki, Finland. e-mail: pirjo.isohanni@helsinki.fi

Manuscript word count: 2904

Abstract word count: 238

Number of figures: 3

Number of tables: 3 


\begin{abstract}
Purpose: To compare the value of serum biomarkers, FGF21 and GDF15 with histological analysis of muscle in the diagnosis of mitochondrial disease.
\end{abstract}

Methods: We collected 194 serum samples from patients with a suspected or known mitochondrial disease. Biomarkers were analyzed blinded using enzyme labelled immunosorbent assay (ELISA). Clinical data was collected using a structured questionnaire.

Results: Only 39\% of patients with genetically verified mitochondrial disease had mitochondrial pathology in their muscle histology. In contrast, biomarkers were elevated in $62 \%$ of patients with genetically verified mitochondrial disease. Those with both biomarkers elevated had a muscle manifesting disorder and a defect affecting mitochondrial DNA expression. If at least one of the biomarkers was induced and the patient had a myopathic disease, a mitochondrial DNA expression disease was the cause with $94 \%$ probability.

Among patients with biomarker analysis and muscle biopsy taken $<12$ months apart, a mitochondrial disorder would have been identified in 70\% with analysis of FGF21 and GDF15 compared to $50 \%$ of patients whom could have been identified with muscle biopsy alone. Muscle findings were non-diagnostic in $72 \%$ (children) and $45 \%$ (adults).

Conclusion: Induction of FGF21 and GDF15 suggest a mitochondrial etiology as an underlying cause of a muscle manifesting disease. Normal biomarker values do not, however, rule out a mitochondrial disorder, especially if the disease does not manifest in muscle. We suggest that FGF21 and GDF15 together should be first-line diagnostic investigations in mitochondrial disease complementing muscle biopsy. 


\section{Synopsis}

Analysis of serum biomarkers should be used in first-line mitochondrial disease diagnostics; they complement but do not entirely remove the need for muscle biopsy. 


\section{Acknowledgments}

We are grateful to the patients and their families for participation in the study. We thank the other members of Mitochondrial Clinical Research Network (Gittan Kollberg, Elsebet Östergaard, Karin Naess, Martin Engvall, Kristoffer Björkman, Charalampos Tzoulis, Kees Schoonderwoerd and late Linda de Meirleir) for valuable discussions.

We thank the following funding sources for support: The Maud Kuistila Memorial Foundation, Emil Aaltonen Foundation, Oskar Öflund Foundation, Waldemar von Frenckells Foundation, Biomedicum Helsinki Foundation, Maire Taponen foundation, Orion Research Foundation, The Finnish Medical Foundation, and Doctoral Program in Biomedicine (DPBM) (JML); The Pediatric Research Foundation (JML, JU and PI); The special governmental subsidy for health sciences research of the Helsinki University Hospital, Academy of Finland (PI and AS); Sigrid Jusélius Foundation, and University of Helsinki (AS); Stichting NeMo (IdC).

\section{Author Contributions}

JML, PI and AS designed the study. JML was responsible for the data collection, analysis of the data and drafted the initial manuscript, and PI and AS contributed to data collection, data analysis, drafting of the manuscript and figures, and approved the final manuscript as submitted. MA, ND, KS, LB, OH, JU, PV, MT, TL and IdC were responsible for data acquisition and analysis, revising the manuscript critically and approving the final manuscript as submitted. All authors are responsible for accuracy and integrity of the work.

\section{Conflicts of Interest}

All the authors declare that they have no conflict of interest. 


\section{Ethics Approval}

The study was approved by The Ethical Review Board of Hospital District of Helsinki and Uusimaa (74/13/03/00/09), other centers obtained approval from their local Ethical Review Boards, and and the study was conducted in accordance with the Helsinki Declaration of 1975, as revised in 2000. All study subjects or their caregivers gave written informed consent.

\section{Keywords}

FGF21, GDF15, muscle biopsy, mitochondrial disease, diagnostics of mitochondrial disease 


\section{Introduction}

Mitochondrial disorders are genetic disorders characterized by defects in oxidative phosporylation which arise from defects in either the mitochondrial or nuclear genome that impair mitochondrial function. ${ }^{1}$ They are progressive diseases often presenting in skeletal muscle, but also affecting organs such as the brain, endocrine organs, liver, heart and sensory organs. The prevalence of these diseases has been estimated to be 1/2000-1/10 000 live births making them among the most common inherited metabolic disorders. ${ }^{2-6}$ Despite involvement of the same intracellular organelle, mitochondrial diseases are highly heterogeneous in both clinical manifestations and genetic basis: currently, more than 250 genes have been identified (The Mitochondrial Disease Sequence Data Resource Consortium, MSeqDR, https://mseqdr.org/).

Despite next generation sequencing (NGS) techniques with improved efficacy of establishing a genetic diagnosis, patients with suspected mitochondrial disease often undergo a muscle biopsy to look for presence of cytochrome c oxidase (COX) deficient fibers or defect in oxidative phosphorylation (OXPHOS) enzyme activities. Other biomarkers, including blood lactate, pyruvate, their ratio and creatine kinase have been used, but these lack both sensitivity and specificity. ${ }^{7}$

Two novel serum biomarkers, fibroblast growth factor 21 (FGF21), ${ }^{7,8}$ and growth differentiation factor $15(\mathrm{GDF} 15)^{9,10}$ have been reported. The efficacy of these biomarkers compared to the traditional biomarkers in diagnosing muscle-manifesting mitochondrial disorders has been replicated in several studies. ${ }^{11-19}$ FGF21 is a metabolic cytokine that is upregulated in the liver 
during fasting to induce lipolysis in adipose tissue of healthy individuals. ${ }^{20}$ GDF15 is a member of the transforming growth factor beta (TGF- $\beta$ ) superfamily, also secreted by the liver, especially in response to liver tissue injury. ${ }^{21}$ In mitochondrial myopathy, which is a genetic disorder leading to OXPHOS defect affecting predominantly, but not exclusively, skeletal muscle, ${ }^{22}$ FGF21 and GDF15 are expressed in skeletal muscle together with OXPHOS defect. ${ }^{7-9}$ In diseased muscle, FGF21 is part of mitochondrial integrated stress response, together with major metabolic remodeling of the tissue. ${ }^{23,24}$ Both cytokines are induced in mice and humans with mitochondrial myopathy, but also in some non-mitochondrial metabolic (FGF21) and degenerative/endstage (GDF15) conditions. ${ }^{25,26}$ However, in the case of FGF21, mitochondrial myopathy is the only condition, in which secretion of FGF21 is directly linked to a monogenic defect. ${ }^{15}$ GDF15, however, has also been reported to be elevated in for example chronic obstructive pulmonary disease (COPD),${ }^{27}$ ovarian cancer, ${ }^{28}$ and diabetes.${ }^{29}$ In addition, it has been described to be a predictive biomarker for cardiovascular disease mortality. ${ }^{30}$

We reported recently that both FGF21 and GDF15 are greatly induced in mitochondrial myopathies caused by mitochondrial DNA (mtDNA) expression disorders (mtDNA deletions that remove tRNA genes, mitochondrial maintenance and mitochondrial translation defects), but not in defects directly affecting mitochondrial OXPHOS subunits or respiratory chain complex assembly. ${ }^{15}$ Both biomarkers correlate to muscle involvement. ${ }^{10,13,31}$ Comparative studies of these biomarkers to muscle histology or OXPHOS activity findings are limited, as is our understanding of how these biomarkers respond to mitochondrial disorders affecting the central nervous system. 
The aims of this study were to clarify the performance of FGF21 and GDF15 compared to conventional diagnostic means, muscle histology and biochemical OXPHOS activity measurement in a prospective setting, and to study whether biomarkers could sometimes replace the need for invasive muscle biopsy in the diagnostics of mitochondrial diseases. 


\section{Methods}

\section{Patient data and samples:}

The study was conducted in five North European centers within the Mitochondrial Clinical Research Network between 2009-2016. Participating centers were Finland (Helsinki and Oulu), Sweden (Gothenburg), the Netherlands (Rotterdam) and Norway (Bergen). Detailed clinical data was collected between April 2013 and February 2017 using an electronic questionnaire designed for this study. The clinical data included age, gender, specific clinical features, biochemical testing and histology of the muscle. The data on the genetic etiology was finalized in March 2018. Symptomatic patients with a clinical suspicion of mitochondrial disease were recruited to the study, and 173 patients were enrolled.

Muscle biopsy samples were analyzed as part of the patients' routine diagnostic evaluation, and serum samples were collected for FGF21 and GDF15 analysis. Histological analysis of the muscle alone or with biochemical analysis was performed based on each center's diagnostic pathway. Genetic studies were performed in each center by their routine diagnostic pathway. Figure 1 summarizes the patient categorization flowchart. Samples were considered as prospective when taken prior to the establishment of a genetic diagnosis. Of the 173 prospective patients, $46(26 \%)$ received a genetic and $22(13 \%)$ a non-genetic diagnosis during the study period while 105 patients $(61 \%)$ remained without specific diagnosis. Twenty-one patients were also included retrospectively. They were patients, who had had a clinical suspicion of mitochondrial disease, and their genetic diagnosis had been identified (Figure 1). The age of the patients varied from 0 to 79 years, of which $45 \%$ were children. There was a slight female 
preponderance (110 females; 84 males). Clinical characteristics are shown in Table 1. Eight patients had diabetes, nine had cardiac and none had renal manifestation.

The FGF21 and GDF15 values were expected to reflect best the skeletal muscle pathology when the time between muscle and serum sampling was not more than 6 (children) or 12 (adults) months. In 68 patients, muscle biopsy was either not taken, or the time elapsed between serum sampling and muscle biopsy was >6-12 months, excluding proper comparison of the muscle disease severity and the biomarker concentrations. Since small amounts of cytochrome-c-oxidase (COX, OXPHOS complex IV) negative fibers are known to accumulate with age, we considered a finding of $<1 \%$ of COX-negative fibers in people over 50 years of age not to be associated with mitochondrial pathology.

The muscle data collected for this study included presence of COX deficient fibers and ragged red fibers, the typical findings of mitochondrial myopathies, as well as the amount of glycogen and lipids, description of histology and suggested diagnosis. OXPHOS analysis was classified abnormal if the activity of enzyme complexes, oxygen consumption (polarography) and/or ATP synthesis activities were decreased (below normal range).

\section{Serum biomarker analyses:}

The serum samples were snap-frozen and stored at $-80{ }^{\circ} \mathrm{C}$ before analysis. The biomarkers were analyzed with commercially available ELISA-kits (FGF21: Biovendor, Brno, Czech Republic; the results exceeding the linear range were replicated with the kit of R\&D Systems, Minneapolis, MN. GDF15: R\&D Systems) according to the manufacturers' instructions. The plate 
absorbances were measured using a SpectraMax 190 absorbance microtiter plate reader (Molecular Devices, Sunnyvale, CA).

\section{Statistical analyses:}

Causative mutations involved in a gene that encodes a protein known to be associated with mitochondrial OXPHOS function or structure, were considered mitochondrial diseases. The pediatric cohort consisted of all those subjects sampled before 16 years of age. The odds ratios were calculated using Fisher's exact test. Association of FGF21 values to GDF15 values was done using Spearman's rank correlation analysis. Association was considered significant if the rvalue exceeded 0.5 and two-sided $p$-value was $<0.05$. In this case, a linear regression model was performed and the $\mathrm{R}^{2}$ and $\mathrm{P}$-values for goodness of fit are reported. All statistical analyses were performed with PRISM 8.4.3 (Graph Pad software, La Jolla, CA). 


\section{Results}

We determined serum FGF21 and GDF15 values in altogether 194 patients, of which 88 were children and 106 adults. FGF21 and GDF15 values correlated significantly to each other ( $\mathrm{n}=194$, $\left.\mathrm{r}=0.5, \mathrm{p}<0.0001, \mathrm{R}^{2}=0.09, \mathrm{p}<0.0001\right)$. The cut-off values for both biomarkers were chosen according to our previous study: the $95^{\text {th }}$ percentile of controls (for FGF21 $331 \mathrm{pg} / \mathrm{ml}$ and for GDF15 $1014 \mathrm{pg} / \mathrm{ml}$ ) and $95^{\text {th }}$ percentile of patients with non-mitochondrial myopathies (for FGF21 $591 \mathrm{pg} / \mathrm{ml}$ and for GDF15 $2581 \mathrm{pg} / \mathrm{ml}) .{ }^{14}$ Based on these cut-offs, we determined the following concentration categories: Low (FGF21 $<331 \mathrm{pg} / \mathrm{ml}$ and GDF15 $<1014 \mathrm{pg} / \mathrm{ml}$ ), intermediate (FGF21 331-591 pg/ml and GDF15 1014-2581 pg/ml), and high (FGF21 >591 $\mathrm{pg} / \mathrm{ml}$ and GDF15 >2581 pg/ml).

Forty-two patients had a known genetic cause for their disease (genetically verified mitochondrial disease), and $62 \%(n=26 / 42)$ of these fell into the intermediate or high category (Table 2A), whereas only 19\% ( $\mathrm{n}=9 / 47)$ of patients with non-mitochondrial disease (genetically or clinically) showed such values (Table 2B and Supplementary table 1).

The odds ratio (OR) for having a mtDNA expression disorder was 48 ( $\mathrm{n}=25$, CI 4.368-567.7, $\mathrm{p}=0.001$ ), if at least one of the biomarkers showed intermediate or high concentrations in a (clinically) muscle manifesting disorder. If at least one of the biomarkers was induced (intermediate or high) in a patient with (clinically) myopathic disease, an mtDNA expression disease was the cause with $94 \%$ probability (CI 0.7302-0.9970, $\mathrm{p}<0.01$, positive predictive value). Patients with elevated biomarker values without a verified genetic diagnosis showed 
more symptoms and findings suggestive of mitochondrial myopathy (progressive external ophthalmoplegia, COX deficient fibers, OXPHOS defect) (Supplementary table 2A) than those who had at least one of the biomarkers in the low range (Supplementary table 2B-C).

Of all 194 patients, 26 patients had both biomarkers elevated, and of those, 50\% had genetically verified mitochondrial disease. 27 patients had a single biomarker elevated, and of those $48 \%$ had genetically verified mitochondrial disease (Table 3 and Supplementary tables 1 and 2).

\section{Findings in patients with genetically verified mitochondrial diseases}

Table 3 summarizes biomarker, clinical and muscle biopsy findings in patients with genetically verified mitochondrial diseases of whom 13/42 had both elevated GDF15 and FGF21 concentrations. They belonged to the following categories: muscle phenotype (clinical, histopathological or biochemical) or defect affecting mtDNA expression (Table 3A). Eighty-two percent of mitochondrial patients who had clinically manifesting muscle disorder showed elevation of biomarkers, whereas only $65 \%$ of them showed mitochondrial pathology in muscle biopsy sample (no muscle sample from two patients). Forty three percent of patients with a genetically verified mitochondrial disease had low GDF15 and FGF21 concentrations (Table 3C). These patients belonged to the categories: non-myopathic phenotypes, genetic defects affecting OXPHOS subunits or assembly, or genetic defects not directly affecting OXPHOS. There were however three exceptions: two patients had mitochondrial tRNA ${ }^{\text {Leu(UUR) }}$ (MTTL1) mutation $(\mathrm{m} .3243 \mathrm{~A}>\mathrm{G})$ and myopathy, and the third patient had a mutation in mitochondrial methionyl-tRNA formyltransferase (MTFMT) and exercise intolerance. Six patients with non- 
OXPHOS disease (and mutations in SLC19A3, ADD3, AMARC, DNAJC19 and ATP1A3, as well as a deletion in chromosome 20p) had elevated biomarker values (Table 2B).

164 patients with muscle biopsy findings

Altogether 164 patients underwent muscle biopsy. Twenty percent of patients had histological findings suggestive of mitochondrial myopathy, and in these all but one genetically verified disorders were mitochondrial. Sixty-six percent of patients showed no or unspecific changes in muscle histology analysis, and $4 \%$ of samples were excluded due to poor quality. Among patients with genetically verified mitochondrial disorders, $39 \%$ had mitochondrial myopathy based on histological assessment, and 54\% of them remained without diagnostic findings (Figure 2E-G).

127 Patients whose muscle biopsy and serum sample were taken less than 6-12 months apart In patients having muscle biopsy and serum samples been taken $<6-12$ months apart, we compared the biomarker concentrations with muscle histology findings (no OXPHOS activity analysis included) ( $\mathrm{n}=127$, Figure 2A-D). If both biomarkers were induced $(\mathrm{n}=14), 57 \%$ had a diagnostic muscle sample (36\% mitochondrial myopathy, $21 \%$ other disease, eg. inflammatory myopathy). If only FGF21 was induced (intermediate or high, $\mathrm{n}=12$ ), $50 \%$ had normal muscle histology, whereas if only GDF15 was induced ( $\mathrm{n}=11), 64 \%$ of histology findings were normal. Normal biomarkers associated with no diagnostic findings in muscle histology in $74 \%$ (either normal or mild unspecific) (Figure 2D). 
Then we compared the diagnostic performance of the biomarkers versus muscle histology (and OXPHOS enzyme analysis, if available) in those patients $(\mathrm{n}=20)$ who had genetically verified mitochondrial disease and biopsy and biomarker taken $<6-12$ months apart. Seventy percent of these patients (14/20) had FGF21 and/or GDF15 values intermediate or high and would have been identified as a likely to have a mitochondrial disease based on serum biomarker analysis only compared to $50 \%$ of patients who could have been identified with muscle biopsy. Three patients (15\%) would only have been diagnosed by analyzing the muscle [two patients with mutations in mitochondrial ATPase subunit 6 (MTATP6): one with high amount of COX negative fibers, the other with decreased oxygen production in OXPHOS analysis. Third patient had neurogenic changes in histology caused by defect in mitofusin 2 (MFN2)]. Three patients had normal-range biomarker values and the muscle histology was judged as either normal or only non-specific changes.

Figure 3 summarizes the performance of muscle histological analysis in identifying a mitochondrial disease [children $(\mathrm{n}=61)$ and adults $(\mathrm{n}=66)$; muscle and serum sample taken less than 6-12 months apart]. A total of 44 children (72\%) had no or mild unspecific changes in their muscle even though four of them had genetically verified mitochondrial disorder (Figure 3A). In adults, histological analysis showed no or mild unspecific findings and the OXPHOS analysis was normal in 30 (45\%) of whom four had a genetically verified mitochondrial disorder (Figure 3B). Taken together, 58\% of patients in this prospective cohort had no clear changes in the muscle histological or biochemical analysis. Of the patients with pathological muscle histology findings ( $n=53$ ), 34\% were considered to have a non-mitochondrial disease, but two were actually later found to have mitochondrial diseases (mutations in MFN2 and MT-TH). Thirty- 
five patients were evaluated to have histological findings suggestive for a mitochondrial disorder, of whom 14 got a genetic diagnosis, 10 were indeed verified to be mitochondrial and four non-mitochondrial diseases (Figure 3A-B). Some of these genetic diagnoses (mutations in SARS2, MT-ATP6 and MT-TL1) were also found in patients whose muscle biopsy sample showed no evident changes. 


\section{Discussion}

We studied 194 patients with a suspected mitochondrial disorder, of whom 173 were investigated prospectively, as part of a routine diagnostic protocol. We compared the diagnostic yield of serum biomarkers FGF21 and GDF15 to the established modalities of routine diagnostic histological and biochemical analysis of muscle. We did not standardize the muscle biopsy analyses but wanted to compare the realistic diagnostic situation to biomarkers FGF21 and GDF15. We found that $58 \%$ of the muscle biopsy samples provided no diagnostic conclusion showing no or only non-specific changes (Figure 3). In contrast, analysis of FGF21 and GDF15 identified $62 \%$ of patients with genetically verified mitochondrial disease, and $82 \%$ of those with muscle manifesting mitochondrial disease. Of the patients with mitochondrial diseases, who had no diagnostic findings in the muscle sample, elevation of the serum biomarkers pointed to mitochondrial disease in $69 \%$ patients. Only four patients had normal biomarker values, despite having mitochondrial abnormalities in their muscle biopsy (Table 3C).

This prospective cohort study confirmed that the induction of FGF21 and GDF15 is highly restricted to muscle-manifesting disorders caused by defects in mtDNA expression. ${ }^{15}$ Pure mitochondrial encephalopathies without muscle involvement usually showed normal values. Our cohort included mitochondrial diseases in which these biomarkers have not previously been studied. These included - mitochondrial aspartyl-tRNA synthetase deficiency/leukoencephalopathy with brain stem and spinal cord involvement and lactate elevation (DARS2); mitochondrial DNA depletion syndrome 13 (FBXL4); axonal, type 2A2 
Charcot-Marie-Tooth disease (MFN2); combined oxidative phosphorylation deficiency 15 (MTFMT); mitochondrial complex I deficiency (NDUFV2), and spastic paraplegia 7 (SPG7)

Our prospective data showed that induction of biomarkers FGF21 and GDF15 clusters to patients with symptoms and findings typical for mitochondrial disorders (Supplementary tables 1 and 2). Previously, out of a total of 69 patients with both biomarkers analyzed, ${ }^{15-17}$ only two patients with a non-mitochondrial disorder had induction of both biomarkers (one with inclusion body myositis (IBM) and another with myotonic dystrophy type II). One patient with Alagille syndrome (deletion in chromosome 20p12.1-p11.23) had both biomarkers highly induced, which may be due to the large chromosomal aberrations including mitochondrial-targeted genes or to be secondary to severe cardiac disease. Overall, the findings are consistent with GDF15 and FGF21 having high specificity to mitochondrial disease group.

Our findings showed that in more than half of the muscle biopsies taken, the histological or OXPHOS activity analysis had no clear diagnostic value, particularly in children. If we had standardized the muscle evaluation and evaluated both histology and biochemistry thoroughly in every patient, the diagnostic yield could have been somewhat bigger but we wanted to include the authentic reports from the patients in this prospective study, and the results indicate the difficulty of mitochondrial diagnoses. Sometimes the findings are not completely logical, and genetic findings are not always easy to interpret. Mitochondrial disease diagnosis is challenging, the analysis of skeletal muscle is not standardized in different laboratories, and biochemical analyses of OXPHOS even less. This is why more biomarkers that are straight forward to do in 
different, even not specialized laboratories, are required, such as serum ELISA analysis of FGF21 and GDF15.

Induction of FGF21 and GDF15 was highly restricted to muscle-manifesting mitochondrial diseases caused by mitochondrial translation defect or mtDNA deletions. FGF21 and GDF15 have good positive predictive values for these disorders, direct further diagnostic procedures, are inexpensive to measure, and the risk of complications is minor compared to surgical muscle biopsy. We therefore propose that the serum biomarkers FGF21 and GDF15 together be used as first-line diagnostic tools in patients with muscle involvement, but can be used in all patients with a suspicion of mitochondrial disease, although in pure encephalopathies biomarkers often remain normal. Normal biomarker values do not, rule out a mitochondrial disorder, especially if the disease does not manifest in muscle, but if at least one of biomarkers is elevated, the next diagnostic examination could be next-generation sequencing analysis of mitochondrial disease genes, both mitochondrial and nuclear DNA. This approach would speed up the diagnostic rate of mitochondrial diseases, bring the diagnostic modalities to everyday use, as well as reduce the need of invasive muscle biopsies, and minimizing the risk of complications. Further, biomarkers might be useful in evaluation of genetic findings, for example variants of unknown significance. 


\section{References}

1.Rahman J, Rahman S. Mitochondrial medicine in the omics era. Lancet 2018; 391: 2560-74.

2. Chinnery PF, Johnson MA, Wardell TM, et al. The epidemiology of pathogenic mitochondrial DNA mutations. Ann Neurol. 2000;48(2):188-193.

3. Skladal D, Halliday J, Thorburn DR. Minimum birth prevalence of mitochondrial respiratory chain disorders in children. Brain. 2003;126(Pt 8):1905-1912.

4. Naviaux RK. Developing a systematic approach to the diagnosis and classification of mitochondrial disease. Mitochondrion. 2004;4(5-6):351-361.

5. Uusimaa J, Moilanen JS, Vainionpää L, et al. Prevalence, segregation, and phenotype of the mitochondrial DNA 3243A>G mutation in children. Ann Neurol. 2007;62(3):278-287.

6. Gorman GS, Schaefer AM, Ng Y, et al. Prevalence of nuclear and mitochondrial DNA mutations related to adult mitochondrial disease. Ann Neurol. 2015;77(5):753-759.

7. Suomalainen A, Elo JM, Pietiläinen KH, et al. FGF-21 as a biomarker for muscle-manifesting mitochondrial respiratory chain deficiencies: A diagnostic study. Lancet Neurol. 2011;10(9):806818.

8. Tyynismaa H, Carroll CJ, Raimundo N, et al. Mitochondrial myopathy induces a starvationlike response. Hum Mol Genet. 2010;19(20):3948-3958.

9. Kalko SG, Paco S, Jou C, et al. Transcriptomic profiling of TK2 deficient human skeletal muscle suggests a role for the p53 signalling pathway and identifies growth and differentiation 
factor-15 as a potential novel biomarker for mitochondrial myopathies. BMC Genomics. 2014;15:91.

10. Yatsuga S, Fujita Y, Ishii A, et al. Growth differentiation factor 15 as a useful biomarker for mitochondrial disorders. Ann Neurol. 2015;78(5):814-823.

11. Davis RL, Liang C, Edema-Hildebrand F, et al. Fibroblast growth factor 21 is a sensitive biomarker of mitochondrial disease. Neurology. 2013;81(21):1819-1826.

12. Salehi MH, Kamalidehghan B, Houshmand M, et al. Association of fibroblast growth factor (FGF-21) as a biomarker with primary mitochondrial disorders, but not with secondary mitochondrial disorders (friedreich ataxia). Mol Biol Rep. 2013;40(11):6495-6499.

13. Koene S, de Laat $\mathrm{P}$, van Tienoven DH, et al. Serum FGF21 levels in adult m.3243A>G carriers: Clinical implications. Neurology. 2014;83(2):125-133.

14. Fujita Y, Ito M, Kojima T, et al. GDF15 is a novel biomarker to evaluate efficacy of pyruvate therapy for mitochondrial diseases. Mitochondrion. 2015;20:34-42.

15. Lehtonen JM, Forsström S, Bottani E, et al. FGF21 is a biomarker for mitochondrial translation and mtDNA maintenance disorders. Neurology. 2016;87(22):2290-2299.

16. Montero R, Yubero D, Villarroya J, et al. GDF-15 is elevated in children with mitochondrial diseases and is induced by mitochondrial dysfunction. PLOS ONE. 2016;11(2):e0148709.

17. Ji X, Zhao L, Ji K, et al. Growth differentiation factor 15 is a novel diagnostic biomarker of mitochondrial diseases. Mol Neurobiol. 2017;54(10):8110-8116. 
18. Lovadi E, Csereklyei M, Merkli H, et al. Elevated FGF 21 in myotonic dystrophy type 1 and mitochondrial diseases. Muscle Nerve. 2017;55(4):564-569.

19. Morovat A, Weerasinghe G, Nesbitt V, et al. Use of FGF-21 as a biomarker of mitochondrial disease in clinical practice. J Clin Med. 2017;6(8).

20. Reitman ML. FGF21: A missing link in the biology of fasting. Cell Metab. 2007;5(6):405407. Accessed Feb 8, 2019.

21. Hsiao EC, Koniaris LG, Zimmers-Koniaris T, et al. Characterization of growthdifferentiation factor 15 , a transforming growth factor beta superfamily member induced following liver injury. Mol Cell Biol. 2000;20(10):3742-3751.

22. Mancuso M, McFarland R, Klopstock T, Hirano M. International workshop: Outcome measures and clinical trial readiness in primary mitochondrial myopathies in children and adults. consensus recommendations. 16-18 november 2016, rome, italy. Neuromuscul Disord. 2017;27(12):1126-1137.

23. Nikkanen J, Forsström S, Euro L, et al. Mitochondrial DNA replication defects disturb cellular dNTP pools and remodel one-carbon metabolism. Cell Metab. 2016;23(4):635-648.

24. Khan NA, Nikkanen J, Yatsuga S, et al. mTORC1 regulates mitochondrial integrated stress response and mitochondrial myopathy progression. Cell Metab. 2017;26(2):428.e5.

25. Zhang X, Yeung DCY, Karpisek M, et al. Serum FGF21 levels are increased in obesity and are independently associated with the metabolic syndrome in humans. Diabetes. 2008;57(5):1246-1253. 
26. Mraz M, Bartlova M, Lacinova Z, et al. Serum concentrations and tissue expression of a novel endocrine regulator fibroblast growth factor-21 in patients with type 2 diabetes and obesity. Clin Endocrinol (Oxf). 2009;71(3):369-375.

27. Mutlu LC, Altintas N, Aydin M, et al. Growth differentiation factor-15 is a novel biomarker predicting acute exacerbation of chronic obstructive pulmonary disease. Inflammation. 2015;38(5):1805-1813.

28. Staff AC, Bock AJ, Becker C, et al. Growth differentiation factor-15 as a prognostic biomarker in ovarian cancer. Gynecol Oncol. 2010;118(3):237-243.

29. Berezin AE. Diabetes mellitus related biomarker: The predictive role of growthdifferentiation factor-15. Diabetes Metab Syndr. 2016;10(1 Suppl 1):154.

30. Rohatgi A, Patel P, Das SR, et al. Association of growth differentiation factor-15 with coronary atherosclerosis and mortality in a young, multiethnic population: Observations from the dallas heart study. Clin Chem. 2012;58(1):172-182.

31. Koene S, de Laat P, van Tienoven DH, et al. Serum GDF15 levels correlate to mitochondrial disease severity and myocardial strain, but not to disease progression in adult m.3243A $>\mathrm{G}$ carriers. JIMD Rep. 2015;24:69-81. 


\section{Figure legends}

Figure 1. Patient categorization flowchart. 194 patients participated to the study, of which 173 were studied prospectively. Numbers indicate the number of patients. Abbreviations: $\mathrm{dg}=$ diagnosis; $\mathrm{mo}=$ months; mito= mitochondrial disease; non-mito $=$ nonmitochondrial disease.

Figure 2. Findings of muscle biopsy sample compared to serum biomarkers (A-D) and genetic etiology. Correlation of biomarker values to muscle biopsy findings in 127 patients (AD) with muscle sample taken close (<6-12 months apart) to the serum sampling. Induction of biomarker equals to FGF21 >331pg/ml and GDF15 >1014pg/ml. Histopathological findings of muscle biopsy samples in all 164 biopsied patients (E) of which 33 had genetically verified mitochondrial (F) and 20 non-mitochondrial $(\mathrm{G})$ disease.

Figure 3. Matching of the genetic etiology to findings of muscle biopsy sample and OXPHOS analysis in those 127 patients, whose muscle biopsy and serum sampling were taken <6-12 months apart. A: Pediatric patients (age at biopsy <16 years), B: Adult patients (age at biopsy $\geq 16$ years).

Abbreviations: $\mathrm{HPD}=$ histopathological diagnosis, $\mathrm{OXPHOS}=$ oxidative phosphorylation, $\mathrm{dg}=$ diagnosis, mito= mitochondrial disorder, non-mito= non-mitochondrial disorder, $\mathrm{MM}=$ mitochondrial myopathy, $*=$ pathogenicity unverified. 
Table 1. Clinical characteristics of the 194 patients in this study.

\begin{tabular}{|l|cc|}
\hline Characteristic & $\begin{array}{l}\text { Number of } \\
\text { patients } \\
\text { (percent) }\end{array}$ \\
\hline $\begin{array}{l}\text { Age at study (years) } \\
<1\end{array}$ & 17 & $(9)$ \\
$1-5$ & 37 & $(19)$ \\
$6-11$ & 22 & $(11)$ \\
$12-15$ & 12 & $(6)$ \\
$\geq 16$ (considered as adults in this study) & 106 & $(55)$ \\
\hline Sex & & \\
Male & 84 & $(43)$ \\
Female & 110 & $(57)$ \\
& & \\
\hline Clinical diagnosis & & \\
Myopathy & 82 & $(42)$ \\
(PEO 6, generalized myopathy 76) & & \\
Encephalo(myo)pathy (Leigh, Alpers, MELAS $\quad$ etc.) & 70 & $(36)$ \\
Ataxia (incl. IOSCA) & 17 & $(8)$ \\
Peripheral neuropathy & 9 & $(5)$ \\
Multi-organ disease & 7 & $(4)$ \\
Cardiomyopathy & 2 & $(1)$ \\
Other (incl. MIDD) & 7 & $(4)$ \\
& & \\
\hline Disease with diabetes & 8 & $(4)$ \\
\hline Disease with cardiac manifestation & 9 & $(5)$ \\
\hline Disease with renal manifestation & - & \\
\hline Disease with liver manifestation & 5 & $(3)$ \\
\hline
\end{tabular}


Table 2. Categorization of A) mitochondrial and B) non-mitochondrial disease genes and the FGF21 and GDF15 values of the patients. The cut-offs are based on Lehtonen et al 2016, the 95th percentile of control population and 95th percentile of non-mitochondrial myopathies. HGNC (HUGO Gene Nomenclature Committee) approved gene symbols are used. Biomarker concentrations are as $\mathrm{pg} / \mathrm{ml}$. Number in parenthesis indicates number of patients if more than one. One patient had both MT-TV and MT-RNR1 mutations. $\triangle$ mtDNA indicates heteroplasmic single large scale mitochondrial DNA deletion.

\begin{tabular}{|c|c|c|c|}
\hline $2 A$. & $\begin{array}{c}\text { FGF-21 }<331 \\
\text { low }\end{array}$ & $\begin{array}{l}\text { FGF21 331-591 } \\
\text { intermediate }\end{array}$ & $\begin{array}{c}\text { FGF21 >591 } \\
\text { high }\end{array}$ \\
\hline $\begin{array}{l}\text { GDF15 } \\
<1014 \\
\text { low }\end{array}$ & $\begin{array}{c}\text { DARS2 } \\
\text { MFN2 (3) } \\
\text { MT-ATP6 (3) } \\
\text { MT-FMT } \\
\text { MT-TL1 (2) } \\
\text { NDUFV2 } \\
\text { TWNK (4) } \\
\text { SARS2 }\end{array}$ & $\begin{array}{c}\text { POLG } \\
\text { MT-TV \& MT-RNR1 } \\
\text { MT-TL1 }\end{array}$ & POLG \\
\hline $\begin{array}{c}\text { GDF15 } \\
\text { 1014-2581 } \\
\text { intermediate }\end{array}$ & $\begin{array}{l}\text { MT-ND5 } \\
\text { MT-TL1 (2) } \\
\text { POLG (3) } \\
\text { TWNK }\end{array}$ & $\begin{array}{c}\text { POLG } \\
\triangle \mathrm{mtDNA} \\
\text { SPG7 }\end{array}$ & $\begin{array}{l}\triangle \mathrm{mtDNA} \\
\text { MT-TL1 } \\
\text { MT-TH }\end{array}$ \\
\hline $\begin{array}{l}\text { GDF15 } \\
>2581 \\
\text { high }\end{array}$ & $\begin{array}{l}\text { SARS2 } \\
\text { FBXL4 }\end{array}$ & MT-TL1 & $\begin{array}{l}\triangle \mathrm{mtDNA}(2) \\
\text { MT-TK } \\
\text { MT-TL1 (3) }\end{array}$ \\
\hline
\end{tabular}




\begin{tabular}{|c|c|c|c|}
\hline $2 \mathrm{~B}$. & $\begin{array}{c}\text { FGF-21 }<331 \\
\text { low }\end{array}$ & $\begin{array}{l}\text { FGF21 331-591 } \\
\text { intermediate }\end{array}$ & $\begin{array}{c}\text { FGF21 >591 } \\
\text { high }\end{array}$ \\
\hline $\begin{array}{c}\text { GDF15 } \\
<1014 \\
\text { low }\end{array}$ & $\begin{array}{c}\text { ANO5 } \\
\text { ATP1A3 } \\
\text { ATP7A (2) } \\
\text { CLCN1 } \\
\text { CPT1 } \\
\text { DMD } \\
\text { DNAJB6 } \\
\text { FOLR1 } \\
\text { HIBCH } \\
\text { ITPR1 } \\
\text { PDHA1 (2) } \\
\text { RYR1 } \\
\text { SPATA5 } \\
\text { STBXP1 } \\
\text { SQSTM1 } \\
\text { TMEM126A } \\
\text { ZNHIT3 }\end{array}$ & none & none \\
\hline $\begin{array}{c}\text { GDF15 } \\
\text { 1014-2581 } \\
\text { intermediate }\end{array}$ & $\begin{array}{l}\text { ATP1A3 } \\
\text { SLC19A3 }\end{array}$ & none & $\begin{array}{c}\text { ADD3 } \\
\text { AMACR } \\
\text { DNAJC19 } \\
\end{array}$ \\
\hline $\begin{array}{c}\text { GDF15 } \\
>2581 \\
\text { high }\end{array}$ & none & none & Chr20p deletion \\
\hline
\end{tabular}


Table 3. FGF21, GDF15, age, phenotype, muscle sample findings in patients with genetically verified mitochondrial disease

Clinical myopathy defined as muscle weakness, PEO, ptosis, exercise intolerance or rhabdomyolysis. HGNC (HUGO Gene

Nomenclature Committee) approved gene symbols are used.

3A. Both FGF21 (>331 pg/ml) and GDF15 (>1014 pg/ml) elevated.

\begin{tabular}{|c|c|c|c|c|c|c|c|}
\hline FGF21 & GDF15 & Gene* & $\begin{array}{c}\text { Age } \\
(\text { years })\end{array}$ & Phenotype & $\begin{array}{c}\text { Clinical } \\
\text { myopathy }\end{array}$ & Muscle biopsy & $\begin{array}{c}\mathrm{RC} \\
\text { deficiency }\end{array}$ \\
\hline 2455 & 2761 & $m t D N A$ deletion $(90 \% \mathrm{~m})$ & 21 & PEO & + & COX-negative fibers & na \\
\hline 396 & 1441 & $P O L G$ & 56 & $\begin{array}{c}\text { Mitochondrial spinocerebellar ataxia } \\
\text { (MSCA) }\end{array}$ & + & COX-negative fibers & na \\
\hline 1232 & 4074 & $m t D N A$ deletion $(50 \% \mathrm{~m})$ & 64 & myopathy & + & $\begin{array}{l}\text { "mitochondrial } \\
\text { myopathy" }\end{array}$ & $\mathrm{CI}$ \\
\hline 398 & 4735 & $M T-T L 1(n a)$ & 60 & MELAS & - & fat droplets in EM & - \\
\hline 456 & 1231 & $m t D N A$ deletion $(50 \% \mathrm{~m})$ & 52 & PEO & + & $\begin{array}{l}\text { ragged blue fibers, } \\
\text { increased staining SDH } \\
\text { of the cellborders }\end{array}$ & $\mathrm{CI}, \mathrm{CV}$ \\
\hline 504 & 1328 & SPG7 & 59 & $\begin{array}{l}\text { ataxia, mental deterioration, } \\
\text { deafness, cardiomyopathy }\end{array}$ & - & RRF & $\begin{array}{c}\mathrm{CI}, \mathrm{O} 2 \\
\text { consumption }\end{array}$ \\
\hline 912 & 2152 & $m t D N A$ deletion $(60 \% \mathrm{~m})$ & 60 & PEO & + & $\begin{array}{l}\text { COX-negative fibers, } \\
\text { RRF }\end{array}$ & 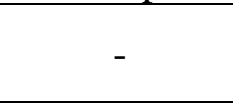 \\
\hline 1956 & 7601 & $M T-T L 1(80 \% \mathrm{~m})$ & 24 & MELAS & + & $\begin{array}{l}\text { abnormal cristae and } \\
\text { mitochondria in EM }\end{array}$ & $\mathrm{CI}$ \\
\hline 878 & 2802 & $M T-T K(93 \% m)$ & 34 & encephalopathy & - & $\mathrm{RRF}$ & $\mathrm{CI}, \mathrm{CV}$ \\
\hline 642 & 1833 & $M T-T L 1(90 \%$ u) & 39 & MELAS & + & normal & na \\
\hline 826 & 2868 & $M T-T L 1(26 \% \mathrm{~m})$ & 30 & myopathy & + & $\begin{array}{l}\text { RRF, abnormal cristae } \\
\text { in EM }\end{array}$ & $\begin{array}{c}\mathrm{CI}, \mathrm{O} 2 \\
\text { consumption }\end{array}$ \\
\hline
\end{tabular}




\begin{tabular}{|c|c|c|c|c|c|c|}
\hline 2170 & 4868 & MT-TL1 $(70 \%, n a)$ & 43 & MELAS/MIDD & + & uninformative \\
\hline 1352 & 1534 & MT-TH $(50 \% \mathrm{~m}, 85 \% \mathrm{~b})$ & 46 & $\begin{array}{c}\text { myopathy, cardiomyopathy, } \\
\text { diabetes, psychiatric disorder }\end{array}$ & $+\begin{array}{c}\text { "necrotising } \\
\text { myopathy" }\end{array}$ & na \\
\hline
\end{tabular}

\section{B. Either FGF21 (>331pg/ml) or GDF15 (>1014pg/ml) elevated.}

\begin{tabular}{|c|c|c|c|c|c|c|c|}
\hline FGF21 & GDF15 & Gene & $\begin{array}{c}\text { Age } \\
\text { (years) }\end{array}$ & Phenotype & $\begin{array}{c}\text { Clinical } \\
\text { myopathy }\end{array}$ & Muscle biopsy & $\begin{array}{c}\mathrm{RC} \\
\text { deficiency }\end{array}$ \\
\hline 628 & 884 & $P O L G$ & 59 & PEO & + & $\begin{array}{l}\text { COX -negative fibers, } \\
\text { mild type } 2 \text { fiber atrophy }\end{array}$ & na \\
\hline 304 & 1074 & $P O L G$ & 41 & $\begin{array}{l}\text { Mitochondrial spinocerebellar } \\
\text { ataxia (MSCA) }\end{array}$ & + & 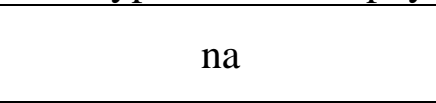 & na \\
\hline 332 & 769 & $P O L G$ & 43 & $\begin{array}{l}\text { Mitochondrial spinocerebellar } \\
\text { ataxia (MSCA) }\end{array}$ & + & na & na \\
\hline 260 & 1362 & $M T-T L 1(19 \% \mathrm{~m})$ & 30 & exercise intolerance, PEO & + & normal & CI \\
\hline 286 & 2074 & $M T-T L 1(62 \% \mathrm{~m})$ & 61 & MELAS & - & $\begin{array}{l}\text { "mitochondrial } \\
\text { myopathy" }\end{array}$ & CIV \\
\hline 262 & 1311 & $P O L G$ & 38 & Alpers syndrome & - & ragged red fibers & $\begin{array}{c}\mathrm{O} 2 \\
\text { consumption }\end{array}$ \\
\hline 408 & 384 & $\begin{array}{c}M T-T V \text { and } M T-R N R l \\
(n a)\end{array}$ & 2 & infantile neuroaxonal dystrophy & - & normal & na \\
\hline 342 & 816 & $M T-T L 1(57 \% \mathrm{~m})$ & 35 & MELAS & + & $\begin{array}{l}\text { "mitochondrial } \\
\text { myopathy" }\end{array}$ & - \\
\hline 284 & 2528 & $P O L G$ & 23 & myopathy & + & unspecific & - \\
\hline 68 & 3208 & $S A R S 2$ & 0 & $\begin{array}{l}\text { encephalomyopathy, congenital } \\
\text { lactic acidosis }\end{array}$ & + & normal & $\begin{array}{l}\text { CI-CV, O2 } \\
\text { consumption }\end{array}$ \\
\hline 198 & 1624 & $M T-N D 5(61 \% m)$ & 2 & Leigh(-like) syndrome & - & normal & $\begin{array}{c}\mathrm{CI}, \mathrm{O} 2 \\
\text { consumption }\end{array}$ \\
\hline
\end{tabular}




\begin{tabular}{|c|c|c|c|c|c|c|c|}
\hline 24 & 2618 & FBXL4 & 0 & cardio-encephalomyopathy & + & normal \\
\hline 96 & 1528 & TWNK & 65 & IOSCA & - & na \\
\hline
\end{tabular}

\section{C. Both FGF21 $(<331 \mathrm{pg} / \mathrm{ml})$ and GDF15 $(<1014 \mathrm{pg} / \mathrm{ml})$ normal.}

\begin{tabular}{|c|c|c|c|c|c|c|c|}
\hline $\begin{array}{c}\text { FGF2 } \\
1\end{array}$ & $\begin{array}{c}\text { GDF1 } \\
5\end{array}$ & Gene & $\begin{array}{c}\begin{array}{c}\text { Age } \\
\text { (years } \\
)\end{array} \\
\end{array}$ & Phenotype & $\begin{array}{c}\text { Clinical } \\
\text { myopath } \\
\mathbf{y} \\
\end{array}$ & Muscle biopsy & $\begin{array}{c}\mathrm{RC} \\
\text { deficiency }\end{array}$ \\
\hline 4 & 328 & $D A R S 2$ & 10 & LBSL & na & na & na \\
\hline 260 & 232 & $M F N 2$ & 15 & peripheral neuropathy & + & unspecific & - \\
\hline 44 & 344 & $M F N 2$ & 13 & peripheral neuropathy & - & na & na \\
\hline 44 & 298 & MFN2 & 13 & peripheral neuropathy & - & na & na \\
\hline 239 & 870 & $M T-A T P 6(n a) * *$ & 32 & $\begin{array}{l}\text { cardio-encephalomyopathy, } \\
\text { myelodysplastic syndrome }\end{array}$ & + & $\begin{array}{c}\text { MM, COX-negative fibers } \\
(>50 \%)\end{array}$ & $\begin{array}{c}\text { CIV, O2 } \\
\text { consumption }\end{array}$ \\
\hline 74 & 348 & $M T-A T P 6(100 \% \mathrm{~m})$ & 3 & NARP / Leigh & - & unspecific & - \\
\hline 160 & 624 & MT-ATP6 (95\% m, 96\% f) & 1 & failure to thrive, poor growth & - & unspecific & $\begin{array}{c}\text { ATP } \\
\text { production }\end{array}$ \\
\hline 244 & 294 & $M T-F M T(n a)$ & 21 & Leigh-like encephalomyopathy & + & na & CI, CIII \\
\hline 258 & 566 & $M T-T L 1(28 \% \mathrm{~m})$ & 33 & MELAS & - & COX-negative fibers, RRF & na \\
\hline 234 & 784 & $M T-T L 1(10 \% \mathrm{~m})$ & 53 & MELAS & + & normal & - \\
\hline 202 & 326 & $N D U F V 2$ & 11 & Leigh syndrome & - & na & na \\
\hline
\end{tabular}




\begin{tabular}{|c|c|c|c|c|c|c|c|}
\hline 98 & 289 & SARS2 & 9 & encephalopathy & - & unspecific \\
\hline 270 & 278 & $T W N K$ & 31 & IOSCA & na & na \\
\hline 120 & 559 & $T W N K$ & 38 & 41 & IOSCA & - & na \\
\hline 163 & 716 & $T W N K$ & 2 & IOSCA & IOSCA & na \\
\hline 190 & 323 & $T W N K$ & na & & na \\
\hline
\end{tabular}

*For mitochondrial DNA mutations, the amount of mutant mitochondrial DNA (per cent) and the tissue studied are in parentheses. Abbreviations: $b=$ blood; $C F T D=$ congenital fiber type disproportion; $C O X=$ cytochrome $c$ oxidase; $f=$ fibroblasts; IOSCA= infantileonset spinocerebellar ataxia; $\mathrm{m}=$ muscle; $\mathrm{MELAS}=$ mitochondrial encephalomyopathy with lactic acidosis and stroke-like episodes; $\mathrm{NARP}=$ neurogenic ataxia and retinitis pigmentosa; $\mathrm{LBSL}=$ leucoencephalopathy with brainstem and spinal cord involvement and lactate elevation; $\mathrm{MM}=$ mitochondrial myopathy; na= not available; $\mathrm{RC}$ deficiency= respiratory chain deficiency (in muscle); $\mathrm{RRF}=$ ragged red fibers; $\mathrm{u}=$ urine; += yes, - = no. ** Novel mtATP6 mutation, unverified. 